\title{
1 Gang der Untersuchung
}

Durch die derzeitige Zinspolitik sind Baufinanzierungsdarlehen zu günstigen Konditionen erhältlich. Die Nachfrage nach Bauland ist entsprechend hoch. Ausgangspunkt ist die angespannte Wohnraumsituation, die nicht nur in Großstädten wie Berlin oder München vorhanden ist. Auch in unserer Region, in Braunschweig oder Hannover, ist die Nachfrage nach bezahlbarem Bauland hoch. Es stellt sich die Frage, ob das Erbbaurecht eine vorteilhafte Alternative zum Grundstückskauf sein kann. Ausgehend von Artikeln unterschiedlicher Onlinezeitschriften ${ }^{1}$ wird zudem der Frage nachgegangen, ob die Anpassung des Erbbauzinses für beide Parteien gerecht erfolgt. Um auf die Zukunftsfähigkeit einzugehen, wird im zweiten Kapitel zunächst grundlegend erläutert wie das Erbbaurecht historisch gewachsen ist. Es erfolgt daraufhin eine rechtliche Einordnung sowie eine Darstellung der rechtlichen und vertraglichen Besonderheiten. Neben dem Erbbauzins wird innerhalb der Arbeit der Fokus zudem auf die Wertsicherungsklauseln gelegt.

Anhand praxisnaher Fallbeispiele zeigt das dritte Kapitel auf, wie bei der derzeitigen Zinslage die Kostenbelastung zweier Familien verläuft. Im Vergleich erfolgt einerseits die Finanzierung des Grundstücks über ein Annuitätendarlehen als auch über die Bestellung eines Erbbaurechts. Ergänzend dazu wird die Änderung der Raten und Gesamtsummen des Erbbaurechts bei einer angenommenen linearen Anpassung des Erbbauzinses am Verbraucherpreisindex (VPI) vorgestellt. Daneben wird ein möglicher Vorteil für Familien mit Kindern herausgearbeitet, welche Ermäßigungen auf den Erbbauzins erhalten. Im vierten Kapitel werden wirtschaftliche, soziale sowie rechtliche Problematiken vorgestellt und diskutiert. Insgesamt bezieht sich die Arbeit vor allem auf die privatrechtliche Seite.

\footnotetext{
${ }^{1}$ Vgl. Seith, Erbbau-Abzocke: Pächter rebellieren gegen Kirchen-Stiftung; Simon/Wenzl, Erbbaurecht, Bodenlose Zahlung.

(C) Springer Fachmedien Wiesbaden 2016 